\title{
Confinement of non-neutral plasmas in the Columbia Non-neutral Torus Experiment
}

\author{
Thomas Sunn Pedersen*, Jason P. Kremer* and Allen H. Boozer* \\ ${ }^{*}$ Columbia University, New York, NY 10027
}

\begin{abstract}
The physics of non-neutral plasmas confined on magnetic surfaces is discussed. The Columbia Non-neutral Torus (CNT), a table-top ultrahigh vacuum stellarator being constructed at Columbia University, is being built to systematically study non-neutral plasmas confined on magnetic surfaces. The experimental design is discussed in the context of relevant physics parameters, such as the number of Debye lengths in the device, and the parallel versus perpendicular time scales.
\end{abstract}

\section{INTRODUCTION}

Confinement systems that use magnetic field lines alone have several advantages over those that use magnetic and electric fields, such as the Penning trap, including the ability to confine positive and negative particles simultaneously, and the ability to confine light, energetic particles. Closed toroidal field line systems have been used to confine pure electron plasmas [1, 2, 3, 4], and more recently, magnetic surface configurations have become of interest as confinement devices for non-neutral plasmas [5, 6]. The physics of pure electron plasmas confined on magnetic surfaces is fundamentally different from previously studied configurations. A magnetic surface configuration is characterized by magnetic field lines that lie on toroidal surfaces (the magnetic surfaces), with each field line coming arbitrarily close to any point on the surface that it lies on. The parallel dynamics of the plasma then determines not just what happens on an isolated field line, but an entire surface. Magnetic surface configurations are well-known in fusion science, in particular the tokamak and the stellarator. A stellarator is a magnetic surface configuration created entirely from magnets external to the plasma, so it has the advantage that it does not require any plasma currents, and can be steady state. A stellarator, the Columbia Non-neutral Torus (CNT) is currently being constructed specifically to investigate the physics of non-neutral plasmas confined on magnetic surfaces. This paper reviews the theory of non-neutral plasmas confined on magnetic surfaces, and discusses some of the experimental parameters that are of importance to a non-neutral stellarator experiment. The CNT experiment is discussed in the context of these parameters. 


\section{CONFINEMENT OF PURE ELECTRON PLASMAS}

\section{Equilibrium}

The equilibrium of a pure electron plasma in a magnetic surface configuration is described by a self-consistent equation for the electrostatic potential [6]:

$$
\varepsilon_{0} \nabla^{2} \phi=e N(\psi) \exp \left(e \phi / T_{e}(\psi)\right)
$$

Here $\psi$ is the magnetic surface coordinate, that is, each magnetic surface is described by $\psi=$ constant . The temperature is taken to be constant on a magnetic surface due to rapid thermalization along field lines, $T_{e}=T_{e}(\psi)$. The function $N(\psi)$ indirectly specifies the density profile. The equilibrium plasma flow is:

$$
\mathbf{v}_{e}=\frac{\left(\nabla p / e n_{e}-\nabla \phi\right) \times \mathbf{B}}{B^{2}}+\mathrm{v}_{\|} \frac{\mathbf{B}}{B}
$$

It can be shown that this flow cannot cross the magnetic surfaces [6]. The parallel flow adjusts itself to make the total particle flux divergence free, even if the perpendicular particle flux is not. With closed toroidal field lines, or in a Penning trap, the parallel flow cannot do this, and hence, contours of constant density and electrostatic potential must coincide in order to keep the perpendicular particle flux divergence free.

\section{Stability}

The equilibrium electrostatic potential given by Equation 1 minimizes an energy-like quantity [7]:

$$
U=\int\left(\frac{1}{2} \varepsilon_{0}(\nabla \phi)^{2}+N(\psi) T_{e}(\psi) \exp \left(e \phi / T_{e}(\psi)\right)\right) d V=\int\left(\frac{1}{2} \varepsilon_{0} E^{2}+p\right) d V
$$

The equilibrium electron density increases near positive image charges. This is in contrast to the Penning trap [8] and the pure toroidal field trap [9], which have electrostatic potentials that maximize the potential energy, and the electron plasma tends to move away from positive image charges. However, the energy-like quantity that is minimized in equilibrium in a magnetic surface configuration is not the free energy, so this does not guarantee stability of all possible configurations. Further work is needed to develop proper stability criteria of pure electron plasmas confined on magnetic surfaces.

\section{Confinement}

Confinement in a non-neutral stellarator is limited by neoclassical diffusion. The guiding center drifts that cause the particles to drift away from the magnetic surfaces are the $E \times B$ drift as well as curvature and $\nabla B$ drifts:

$$
\mathbf{v}_{D}=\frac{\mathbf{E} \times \mathbf{B}}{B^{2}}+\left(\frac{1}{2} m \mathrm{v}_{\perp}^{2}+m \mathrm{v}_{\|}^{2}\right) \frac{\nabla B \times \mathbf{B}}{e B^{3}}
$$


The $E \times B$ drift causes drift excursions away from the magnetic surfaces because the electrostatic potential contours do not coincide exactly with the magnetic surfaces. The electrostatic potential contours do match very closely to the magnetic surfaces in any region with appreciable plasma density, unless the Debye length is large. A simple scaling estimate yields the following particle confinement time.

$$
\tau_{p} \approx \tau_{e} \frac{a^{4}}{\lambda_{D}^{4}}
$$

Here $\tau_{e}$ is the electron collision time, and the estimate above is valid for small Debye lengths.

\section{Initial plasma formation in a stellarator}

Equation 5 suggests that single particles injected into an empty stellarator will not be confined. However, the expression above ignores the confinement due to the magnetic surfaces themselves, which provide confinement for $a / \lambda_{D} \rightarrow 0$. In a classical stellarator, such as CNT, there will be two kinds of particles, well-confined and poorly confined particles (particles on bad orbits). Generally, passing particles are well-confined with very long confinement times. The mirror-trapped poorly confined particles will be confined for at least $\tau \approx a / \mathrm{v}_{D}$, where $a$ is the minor radius and $\mathrm{v}_{D}$ is the guiding center drift velocity, Equation 4. Even though this confinement time is low, it is sufficient to start the accumulation of electrons and establish a finite Debye length. In addition, the well-confined particles will be confined much longer. Hence, the confinement of the magnetic surfaces will allow initial accumulation of electrons even in the zero density limit, and confinement will improve as the number of Debye lengths increases. It should be mentioned that quasi-symmetric stellarators, which have specially optimized magnetic surfaces, the fraction of poorly confined particles is essentially zero, and neoclassical confinement times are very long in the complete absence of space charge. Such stellarators are generally characterized by having rather complicated coils.

\section{CONFINEMENT OF PARTLY NEUTRALIZED AND ELECTRON-POSITRON PLASMAS}

A stellarator confines both positive and negative particles simultaneously whether space charge and internal currents are present or not. This allows the study of plasmas of arbitrary neutralization, a field of plasma physics that is currently largely unexplored. Positive particles, ions or positrons, will be very well confined in an electron-rich plasma, by the overall negative space charge as well as by the magnetic surfaces. As one slowly neutralizes the plasma, the electric field weakens, as the density rises. The electron confinement time will then be [10]:

$$
\tau_{p} \approx \tau_{e} \frac{a^{4}}{\lambda_{C}^{4}}
$$


This is a similar scaling to that of a pure electron plasma, however, the Debye length $\lambda_{D}$ is replaced by the Coulomb length,

$$
\lambda_{C}^{2}=\frac{\left|n_{e}-n_{p}\right|}{n_{e}+n_{p}} \lambda_{D}^{2}
$$

Here, $n_{e}$ is the electron density and $n_{p}$ is the density of the positive species, assumed to be a proton or a positron. In a quasi-neutral plasma $a / \lambda_{C}$ is on the order of 1 or less, whereas in a pure electron plasma, $a / \lambda_{C}=a / \lambda_{D} \gg 1$. A partly neutralized plasma may be characterized by $a / \lambda_{D}>a / \lambda_{C} \gg 1$. The confinement time given by Equation 6 is long in this limit, and that may allow significant accumulation of positrons injected into a stellarator containing an initially pure electron plasma, even with the relatively weak positron sources available today [10]. Hence, this may be an attractive way to create the first laboratory electron-positron plasma.

\section{DESIGN OF THE COLUMBIA NON-NEUTRAL TORUS}

The Columbia Non-neutral Torus (CNT), a small stellarator currently being constructed at Columbia University, is the first experiment specifically designed to study the physics of non-neutral and electron-positron plasmas confined in a stellarator. The coil configuration is very simple, consisting only of four circular, planar coils. Two of these coils are interlocked and will be in inside the vacuum chamber. The stellarator magnetic field is characterized by $\mathrm{B}=0.2 \mathrm{~T}$ on the magnetic axis, (average) minor radius $a=0.1 \mathrm{~m}$, (average) major radius $R=0.3 \mathrm{~m}$, rotational transform $\imath=0.2-0.6$, and the device is designed to have a neutral base pressure of $<3 \times 10^{-10}$ Torr. $\imath$ is the rotational transform, which is the number of times a magnetic field line winds around poloidally divided by the number of times it winds around toroidally. The magnets will be driven by a $200 \mathrm{~kW}$ DC power supply, and the pulse length will be at least 15 seconds at full field, limited by the allowable temperature rise of the copper conductors.

\section{Important physics parameters}

In order to guide the design of CNT, we have identified important physics parameters for a non-neutral stellarator. Specifically, we have focused on parameters of importance to confining pure electron plasmas. The most fundamental physics parameter of any plasma physics experiment is $a / \lambda_{D}$, where $a$ is the smallest characteristic size of the plasma, in the case of a stellarator, the minor radius. In order for the electron cloud to be a plasma, $a / \lambda_{D} \gg 1$. In a non-neutral plasma experiment, including CNT, this is a non-trivial constraint that requires careful matching of the injected electron energy to the plasma potential, or some method of cooling the plasma after it has been injected. $a / \lambda_{D} \gg 1$ is particularly important in a non-neutral stellarator, given the predicted strong scaling of the confinement time with $a / \lambda_{D}$, Equation 5. Another important parameter is the time scale for ion accumulation due to ionization of background neutrals, $\tau_{i}$. When $\tau_{i} \gg \tau_{p}$, electron plasmas will decay before being significantly contaminated. When 
$\tau_{i} \ll \tau_{e}$, ions will significantly neutralize an initially pure electron plasma before it decays away. It is desirable to maximize both time scales, since either one can trivially be decreased. A large $\tau_{i}$ will be achieved through the ultrahigh vacuum design and operation at low plasma temperatures. A large $\tau_{p}$ can be achieved by making the Debye length short compared to the system size, although there is a tradeoff involved which will be addressed in the following.

A key issue for a non-neutral plasma on magnetic surfaces is whether the plasma truly equilibrates on a magnetic surface through parallel dynamics faster than the $E \times B$ drift can take the plasma away from the magnetic surfaces. In a quasi-neutral plasma, this is basically always true, but the $E \times B$ drift can be very large in non-neutral plasmas. The time scale for perpendicular distortions is the $E \times B$ rotation time $\tau_{\perp}=2 \pi a /(E / B)$. Any breaking of the parallel force balance will lead to plasma oscillations which are subsequently Landau damped in a finite temperature plasma. We approximate the parallel relaxation time by the time it takes a thermal particle to move along the magnetic field to fully explore the magnetic surface, $\left.\tau_{\|}=2 \pi R /\left(l \sqrt{(} T_{e} / m_{e}\right)\right)$. Then in order to ensure that the parallel force balance dominates over diocotron-type perpendicular dynamics, we must have $\tau_{\perp} / \tau_{\|} \gg 1$. The ratio of the two can be expressed as:

$$
\frac{\tau_{\perp}}{\tau_{\|}} \approx 2 \sqrt{2} \frac{a}{R} l \sqrt{\frac{n_{B}}{n_{e}}} \frac{\lambda_{D}}{a}
$$

Here, $n_{B}=\varepsilon_{0} B^{2} / 2 m_{e}$ is the Brillouin density. Since $\imath<1, a / R<1$, the conditions that $a / \lambda_{D} \gg 1$ and $\tau_{\perp} / \tau_{\|} \gg 1$ can only be satisfied simultaneously if $\sqrt{n_{B} / n_{e}} \gg 1$, which is well satisfied in most non-neutral plasma experiments. CNT is designed to operate in this regime as well.

\section{Engineering design optimization}

One of the main design considerations for CNT is the ability to reach ultrahigh vacuum levels. It is particularly important to avoid ion contamination in toroidal pure electron plasma experiments, because the ions will accumulate due to the electrostatic attraction to the electrons. CNT has been designed to reach ultrahigh vacuum levels $\left(<3 \times 10^{-10}\right.$ Torr $)$ in order to allow studies of pure electron plasmas for long times.

CNT was also designed to maximize the ratio $\tau_{\perp} / \tau_{\|}$, Equation 8 . It can be reexpressed as:

$$
\frac{\tau_{\perp}}{\tau_{\|}} \approx 2 \sqrt{2}\left(\varepsilon_{a} \imath B\right)\left(\frac{\lambda_{D}}{a} \sqrt{\frac{\varepsilon_{0}}{2 m_{e} n_{e}}}\right)
$$

Here, we have separated plasma parameters from experimental design parameters, and introduced the inverse aspect ratio, $\varepsilon_{a}=a / R$. It is clear that in order to achieve $a / \lambda_{D} \gg 1$ and $\tau_{\perp} / \tau_{\|} \gg 1$, one should maximize the product $\varepsilon_{a} l B$ of the stellarator magnetic field. CNT was specifically optimized for this ratio. The maximization of $\varepsilon_{a} \imath B$ led to a design in which the two interlocking coils were placed inside the vacuum chamber, despite the extra complexity of having in-vessel, interlocking coils. This allowed us to use a large, 
cylindrical vacuum chamber, instead of a tight-fitting toroidal chamber. The elimination of the tight-fitting toroidal chamber has the following advantages:

- An increased copper cross section, allowing a stronger magnetic field and a longer pulse length with the same power supply.

- Increased plasma minor radius $a$ for the same major radius. The original vacuum chamber actually cut off good magnetic surfaces.

- Relatively large tilt angles became much more easily accommodated, allowing a large increase in $\boldsymbol{t}$.

- The ability to change the angle between the two interlocking coils

The ability to change the tilt angle in the experiment gives tremendous flexibility in changing the magnetic topology. For example, $l$ is decreased by a factor of 3 from 0.6 to 0.2 by decreasing the angle between the interlocking coils from 88 to 64 degrees. Details about the magnetic configuration and field error sensitivity are presented elsewhere [11]. The redesign and reoptimization of the experiment led to an improvement of a factor of 8 in $\varepsilon_{a} l B$ over the previous design which had the interlocking coils external to the plasma. The improvement came in all three variables, $\varepsilon_{a}$ was increased by a factor of $1.3, \imath$ by a factor of 3, and $B$ by a factor of 2 . As shown in the next section, this improves the operating space for CNT significantly.

\section{Physics parameter regimes of $\mathrm{CNT}$}

There are numerous important physics parameters in a non-neutral stellarator. For clarity, we will primarily focus on three key parameters, the number of Debye lengths in the device, $a / \lambda_{D}$, the ratio of perpendicular to parallel dynamical time scale $\tau_{\perp} / \tau_{\|}$ (Eq. 8), and the characteristic time of ion contamination, $\tau_{i}$. Larger values are more desirable for all of these parameters, but as discussed earlier, there is a tradeoff between maximizing $a / \lambda_{D}$ and maximizing $\tau_{\perp} / \tau_{\|}$. In Figure 1, we show contours of constant values of each of these three parameters for the optimized CNT design. It is seen that there is a reasonably large range of densities and temperatures where all three parameters have acceptable values. A desirable operational point could be $n_{e}=10^{12} \mathrm{~m}^{-3}$ and $T_{e}=1$ $\mathrm{eV}$. At this point, $a / \lambda_{D}=13.4, \tau_{\perp} / \tau_{\|}=18.5, \tau_{i}=2.7 \times 10^{5} \mathrm{~s}$. The calculations here assume for simplicity that the neutrals are hydrogen atoms, which is not an unreasonable assumption as hydrogen often dominates in the ultrahigh vacuum range. The electron confinement time is more than one hour as predicted by Equation 5, dominated by electron-electron collisional transport. The ion contamination time is a very strong function of temperature though. At $T_{e}=5 \mathrm{eV}, \tau_{i}=2.4 \mathrm{~s}$, still rather long, but now significantly shorter than the electron confinement time as well as the experimentally achievable pulse length. Finally, in Figure 2, we show the optimized CNT but with a poorer vacuum, $10^{-8}$ Torr. Interesting experiments can still be performed, but unless one operates at very low temperatures $\left(T_{e}<2 \mathrm{eV}\right)$, ion contamination will be a limiting factor. 


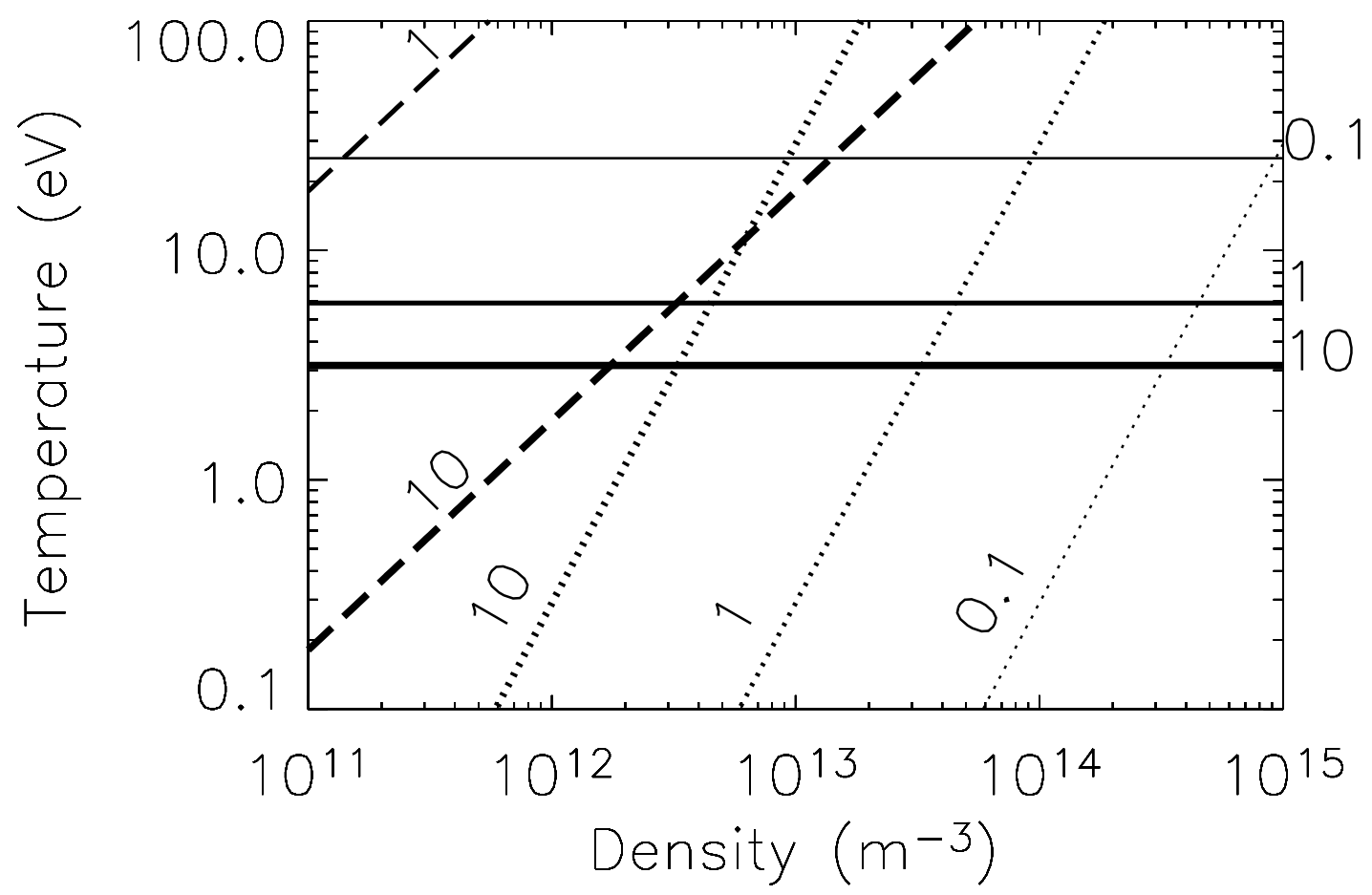

FIGURE 1. Some physics parameters for the optimized design of CNT. Contours of constant $a / \lambda_{D}$ (dashed lines), $\tau_{\perp} / \tau_{\|}$(dotted lines) and $\tau_{i}$ (in seconds, solid lines). The thickest lines indicate a value of 10 , the intermediate lines a value of 1 , and the thinnest lines a value of 0.1

\section{CONCLUSION}

CNT is being constructed specifically to study non-neutral and electron-positron plasmas confined in a stellarator. The design is a compromise between the need to build the device as easily and economically as possible, and the desire to access the most interesting parameter regimes of such a device. We arrived at a design with a simple coil configuration, two adjustable in-vessel coils, a significant magnetic field strength, and an ultrahigh vacuum. The experiment is currently under construction.

\section{ACKNOWLEDGMENTS}

The authors would like to acknowledge the contributions of F. Dahlgren, W. Reiersen and N. Pomphrey on the stellarator design, and W. Dorland on gyrokinetic simulations of electron-positron plasmas. This work was supported by the United States Department of Energy Grant DE-FG02-02ER54690. 


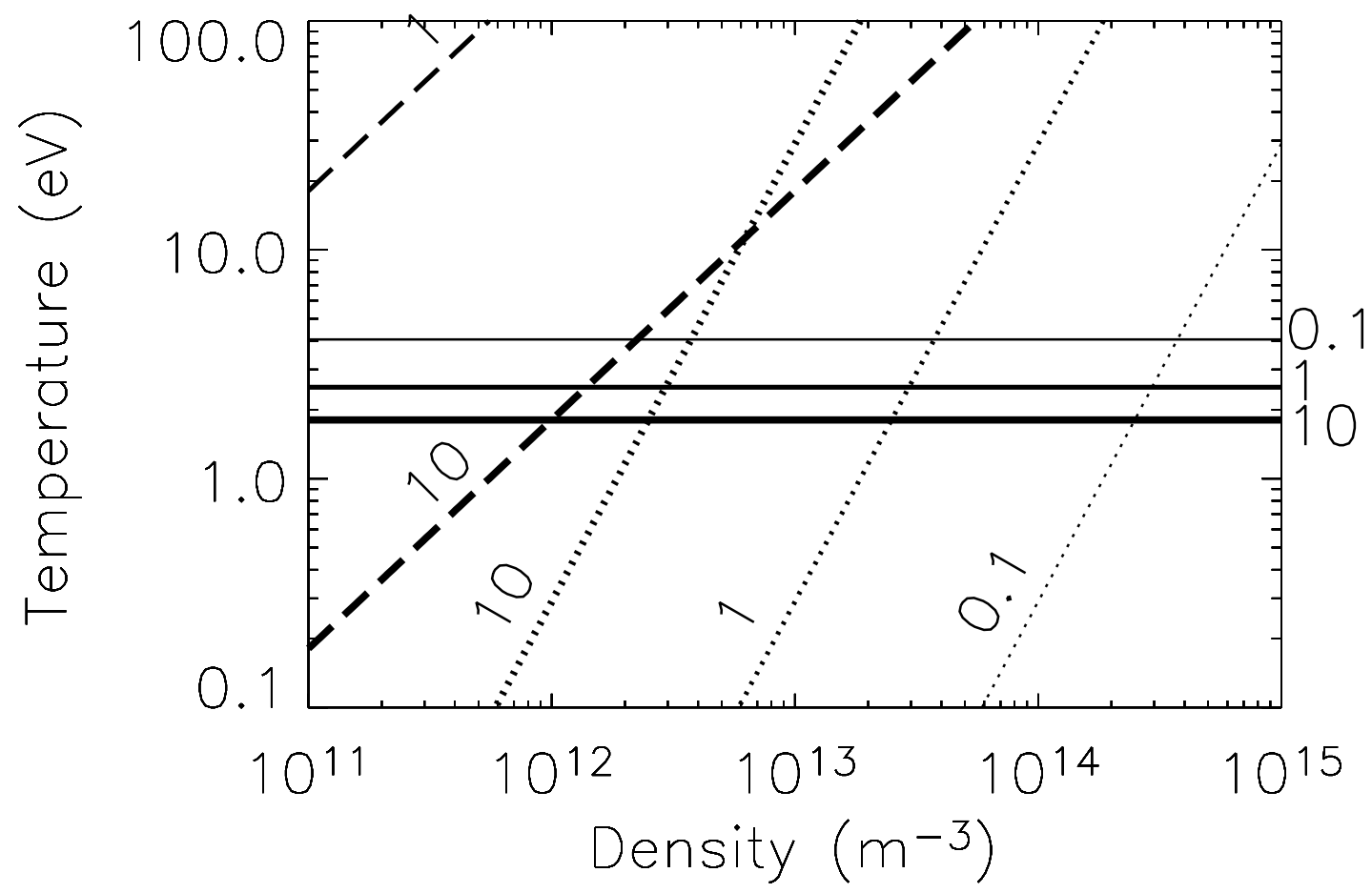

FIGURE 2. Some physics parameters for CNT if the neutral pressure were to be $1 \times 10^{-8}$ Torr. Contours of constant $a / \lambda_{D}$ (dashed lines), $\tau_{\perp} / \tau_{\|}$(dotted lines) and $\tau_{i}$ (in seconds, solid lines). The thickest lines indicate a value of 10 , the intermediate lines a value of 1 , and the thinnest lines a value of 0.1

\section{REFERENCES}

1. Daugherty, J., Eninger, J., and Janes, G., Phys. Fluids, 12, 2677 (1969).

2. Clark, W., Korn, P., Mondelli, A., and Rostoker, N., Phys. Rev. Letters, 37, 592 (1976).

3. Zaveri, P., John, P., Avinash, K., and Kaw, P., Phys. Rev. Letters, 68, 3295 (1992).

4. Stoneking, M. R., Fontana, P., and Sampson, R., Phys. Plasmas, 9, 766 (2002).

5. Yoshida, Z., Ogawa, Y., Morikawa, J., et al., AIP Conf. Proceedings, 498, 397 (1999).

6. Pedersen, T. S., and Boozer, A. H., Phys. Rev. Letters, 88, 205002 (2002).

7. Pedersen, T. S., Phys. Plasmas, 10, 334 (2003).

8. Notte, J., Peurrung, A. J., Fajans, J., Chu, R., and Wurtele, J. S., Phys. Rev. Letters, 69, 3056 (1992).

9. O'Neil, T., and Smith, R., Phys. Plasmas, 1, 2430 (1994).

10. Pedersen, T. S., Boozer, A. H., Dorland, W., Kremer, J. P., and Schmitt, R., J. Phys. B, 36, 1029 (2003).

11. Kremer, J. P., Pedersen, T. S., Pomphrey, N., Reiersen, W., and Dahlgren, F., Non-neutral Plasma Workshop, AIP Proceedings (2003). 